\title{
Detectability of Wolf-Rayet stars in M33 and Beyond the Local Group
}

\author{
Aaron J. Brocklebank, J. L. Pledger and A. E. Sansom \\ Jeremiah Horrocks Institute, University of Central Lancashire, Preston PR1 2HE, UK \\ email: ajbrocklebank1@uclan.ac.uk
}

\begin{abstract}
To understand how complete our surveys of Wolf-Rayet (WR) stars can be with the current generation of telescopes, we study images of M33, a galaxy with a nearly complete WR catalogue, and degrade them to investigate the detectability of WRs out to 30Mpc. We lose almost half of our sample at $4.2 \mathrm{Mpc}$, and at $30 \mathrm{Mpc}$ we detect only those WRs in bright regions.
\end{abstract}

Keywords. stars: Wolf-Rayet, stars: statistics, galaxies: stellar content, galaxies: individual: M33

Wolf-Rayet stars (WRs) are expected to be the progenitors of $\mathrm{H}$ and $\mathrm{H}+\mathrm{He}$ deficient Type Ib and Ic supernovae (SNe). With the aim of directly detecting a SNe Ib/c progenitor, as done with the red supergiant progenitors of Type II-P SNe, surveys of WR stars in nearby galaxies are crucial, some of which have been done already. However, there is an uncertainty as to how complete our surveys beyond the Local Group can be with the current generation of telescopes.

In this study we mimicked observations of WR stars in face-on spiral galaxies at different distances based on a deep WR survey of M33 (Neugent \& Massey 2011). We investigated how many WR stars were able to be detected at distances out to $30 \mathrm{Mpc}$. We also looked specifically at the fraction of WC and WN stars recovered to compare their detectability.

The images were blurred then binned (using IRAF) in order to resemble both the seeing effects and CCD sampling of observations at each mimicked distance (4.2, 8.4, 16.8 and $30.2 \mathrm{Mpc}$ ). Standard photometric techniques were then used to see whether the known WR stars were found to have a helium excess (see Neugent \& Massey 2011 for a description of the filters used).

At $4.2 \mathrm{Mpc}$, only $56 \%$ of WRs are found. At $16.8 \mathrm{Mpc}$, only $23 \%$ of WRs are recovered and $17 \%$ at $30.2 \mathrm{Mpc}$, most of which are in dense, bright stellar regions. Beyond $\sim 10 \mathrm{Mpc}$, the WC stars become notably more difficult to detect than the WNs (19\% and 11\% recovery respectively at $30.2 \mathrm{Mpc}$ ). Using the Fruchter et al. (2006) method to compare the brightness at the location of the WRs with that of the whole galaxy, the distribution shifts to lower brightness and appears to almost follow the galaxy light. At $16.8 \mathrm{Mpc}$ the distribution differs significantly $(3.9 \sigma)$ to that of the original WRs.

\section{References}

Fruchter, A. S., Levan, A. J., Strolger, L., et al. 2006, Nature, 441, 463

Neugent, K. \& Massey, P. 2011, ApJ, 733, 123 